Abstract IDDF2019-ABS-0209 Table 2 Analysis results: 28 tissue-common pathways enriched in six tissues.

\begin{tabular}{|c|c|}
\hline NAME & DATABASE \\
\hline Activation of NF-kappaB in B cells; & reactome \\
\hline Oxygen-dependent proline hydroxylation of Hypoxia-inducible Factor Alpha; & reactome \\
\hline ER-Phagosome pathway; & reactome \\
\hline Cross-presentation of soluble exogenous antigens (endosomes); & reactome \\
\hline Autodegradation of Cdh1 by Cdh1:APC/C; & reactome \\
\hline APC/C:Cdc20 mediated degradation of Securin; & reactome \\
\hline $\begin{array}{l}\text { APC/C:Cdh1 mediated degradation of } \mathrm{Cdc} 20 \text { and other } \mathrm{APC} / \mathrm{C}: \mathrm{Cdh} 1 \text { targeted proteins in late } \\
\text { mitosis/early G1; }\end{array}$ & reactome \\
\hline Cdc20:Phospho-APC/C mediated degradation of Cyclin A; & reactome \\
\hline Vpu mediated degradation of CD4; & reactome \\
\hline SCF(Skp2)-mediated degradation of p27/p21; & reactome \\
\hline Formation of the beta-catenin:TCFtransactivating complex; & reactome \\
\hline Downstream TCR signaling; & reactome \\
\hline DNA Damage/Telomere Stress Induced Senescence; & reactome \\
\hline FCERI mediated NF-kB activation; & reactome \\
\hline Regulation of ornithine decarboxylase (ODC); & reactome \\
\hline AUF1 (hnRNP D0) binds and destabilizes mRNA; & reactome \\
\hline Asymmetric localization of PCP proteins; & reactome \\
\hline Degradation of DVL; & reactome \\
\hline Defective CFTR causes cystic fibrosis; & reactome \\
\hline Retrograde transport at the Trans-Golgi-Network; & reactome \\
\hline CDK-mediated phosphorylation and removal of Cdc6; & reactome \\
\hline Ubiquitin-dependent degradation of Cyclin D1; & reactome \\
\hline G2/M Checkpoints; & reactome \\
\hline The role of GTSE1 in G2/M progression after G2 checkpoint; & reactome \\
\hline FBXL7 down-regulates AURKA during mitotic entry and in early mitosis; & reactome \\
\hline Regulation of PTEN stability and activity; & reactome \\
\hline TGF beta receptor I degradation signaling (TGF-beta_super_family_signaling_pathway(canonical)) & inoh \\
\hline Integrin signaling pathway ( Integrin signaling pathway) & inoh \\
\hline
\end{tabular}

Conclusions Totally, we proposed a new viewpoint as pan-tissue analysis and implemented a pathway-based computational approach to detect the critical difference and common pathways in the responsive tissues during T2D progression, and also shown that the tissue-common pathways can especially serve as genetic warning signals for the T2D sub-states (e.g.complications).

\section{IDDF2019-ABS-0224 SECRETOME MODULATION OF CACO2 CELL LINE INDUCED BY A MULTI-STRAIN PROBIOTIC}

${ }^{1}$ Valentina Petito*, ${ }^{2}$ Viviana Greco, ${ }^{3}$ Lucrezia Laterza, ${ }^{3}$ Cristina Graziani, ${ }^{4}$ Stefano Colombo, ${ }^{3}$ Loris Lopetuso, ${ }^{3}$ Franco Scaldaferri, ${ }^{2}$ Andrea Urbani, ${ }^{3}$ Antonio Gasbarrini. ${ }^{1}$ Institute of Medical Pathology, Universita cattolica del Sacro Cuore, Rome, Italy; ${ }^{2}$ Institute of Biochemistry and Clinical Biochemistry, Universita cattolica del Sacro Cuore, Rome, Italy; ${ }^{3}$ Gastroenterological Area, Gastroenterological, Endocrino-Metabolical AND Nefro-urological Sciences Department, Fondazione Policlinico Universitario Gemelli IRCCS, Rome, Italy; ${ }^{4}$ Beingpharma S.r.l, Milan, Italy

\subsection{6/gutinl-2019-IDDFabstracts.41}

Background Probiotics are defined as live, non-pathogenic bacteria that confer health benefits beyond their nutritional value. Particularly VSL\#3, a probiotic mix containing 4 strains of Lactobacilli (L. paracasei, L. plantarum, L. acidophilus and L. delbrueckii subsp. bulgaricus), 3 strains of Bifidobacteria (B. longum, B. infantis, B. breve) and Streptococcus thermophilus, has demonstrated efficacy in the management of diseases characterized by increased intestinal permeability. The aim of the present study was to study secreted bioactive factors in order to evaluate the mechanisms of action to enhance intestinal epithelia.

Methods Two different lots of VSL\#3 (Manufacturer: Nutrilinea Srl, Gallarate (VA) - Italy, lot \#802097 and lot \#802100) were used. Caco2 cell line were treated with a conditioning media (CM) prepared using $1 \mathrm{~g}$ of probiotic formula grown in D-MEM cell culture medium (free of serum and antibiotics) at $37^{\circ} \mathrm{C}$ for 48 hours without shaking and in anaerobic conditions. Caco 2 were treated with diluted $\mathrm{CM}$ at $1: 10$ and $1: 25$ for 24 and 48 hours. Media culture for each condition has been collected and analyzed by a deeper proteomics approach. Differential protein expression was evaluated by shotgun proteomics analysis based on $\mathrm{nLC}-\mathrm{HDMS}^{\mathrm{E}}$ and carried out on Synapt G2-Si mass spectrometer.

Results The analysis of supernatants from $\mathrm{Caco} 2$, treated with $\mathrm{CM}$, showed the presence of bacteria strain-specific proteins. Human proteins synthesized from $\mathrm{CaCo} 2$ were also identified, such as caspase 1, IL8, HSP70, HSP70b, HSP90, HSP105. The productions were time- and dose-dependent. In CM diluted 1:10, probiotic-derived proteins have been shown to be more expressed at 24 hours. Human caspase 1, IL8, HSP 70, HSP 70b, HSP 90, HSP 105 were also found upregulated in Caco2 treated for 24 hours with CM diluted 1:10.

Conclusions This is the first time where a probiotic secretome was explored. Analysis of secretome from $\mathrm{Caco} 2$ treated with $\mathrm{CM}$ helped us to understand the mechanism by probiotics can enhance intestinal barrier: by strengthen the autophagy process, an arm of innate immunity, by overexpression of caspase 1, IL8 and HSP 70 , and by HSPs dependent modulation of inflammation. 